\title{
Small Vessel Infarcts and Microbleeds Associated with Radiation Exposure
}

\author{
Nandavar Shobha, Eric E. Smith, Andrew M. Demchuk, Nicolas U. Weir
}

Can. J. Neurol. Sci. 2009; 36: 376-378

Radiation induced strokes are classically secondary to the accelerated atherosclerosis of the large vessels of the brain. We describe a patient who presented with recurrent episodes of lacunar strokes without large vessel disease, microbleeds and a single episode of hemorrhagic stroke associated with cranial irradiation.

\section{CASe Description}

Mr. WG, a 51-year-old gentleman was treated with radiotherapy for pontine glioma in 1984. He received $50 \mathrm{~Gy}$ over one month in 16 sittings. He remained asymptomatic until 1994 when, aged 37 years, he had his first stroke which caused a right facial weakness. Computed tomogram (CT) and magnetic resonance image (MRI) brain did not reveal any acute infarct. The CT angiography of the cerebral and cervical vessels was normal. Two years later he re-presented with acute dysarthria and MRI brain scan showed chronic lacunar infarcts in the gangliocapsular regions, but no acute infarcts. In September 2005 , he presented with a right hemiparesis caused by a thalamic hemorrhage on CT (Figure 1). In 2007 he returned with a right hemiparesis and dysphagia, with no acute lesion seen on CT, chronic lacunar infarcts were present. Most recently, in 2008 he presented with a sudden worsening of dysarthria and dysphagia. In addition, over the last ten years he had progressive bilateral hearing loss and recurrent headaches (one to two per month).

He had no past history of hypertension, diabetes mellitus, dyslipidemia or coronary artery disease. There was no family history of strokes. He was a moderate smoker until 2005.

\section{Examination}

Examination revealed a conscious, alert patient with normal comprehension, but severe dysarthria and bulbar dysphonia. Exotropia was present in the right eye and bilateral upgaze was restricted. His fundi were normal. He had bilateral lower motor neuron facial weakness; bilateral sensorineural hearing loss; bilateral reduction in palatal movements and absent gag reflex. There was no atrophy or fasciculation of the tongue, which moved normally. He had a moderate spastic quadriparesis (grade 4/5) with brisk reflexes on the right and bilateral extensor plantar responses. There was incoordination of all four limbs (right greater than left). The sensory examination was normal.

\section{Imaging}

A CT brain during the current presentation did not show any acute infarcts or hemorrhage. The MRI brain revealed chronic lacunar infarcts in the gangliocapsular regions bilaterally, and

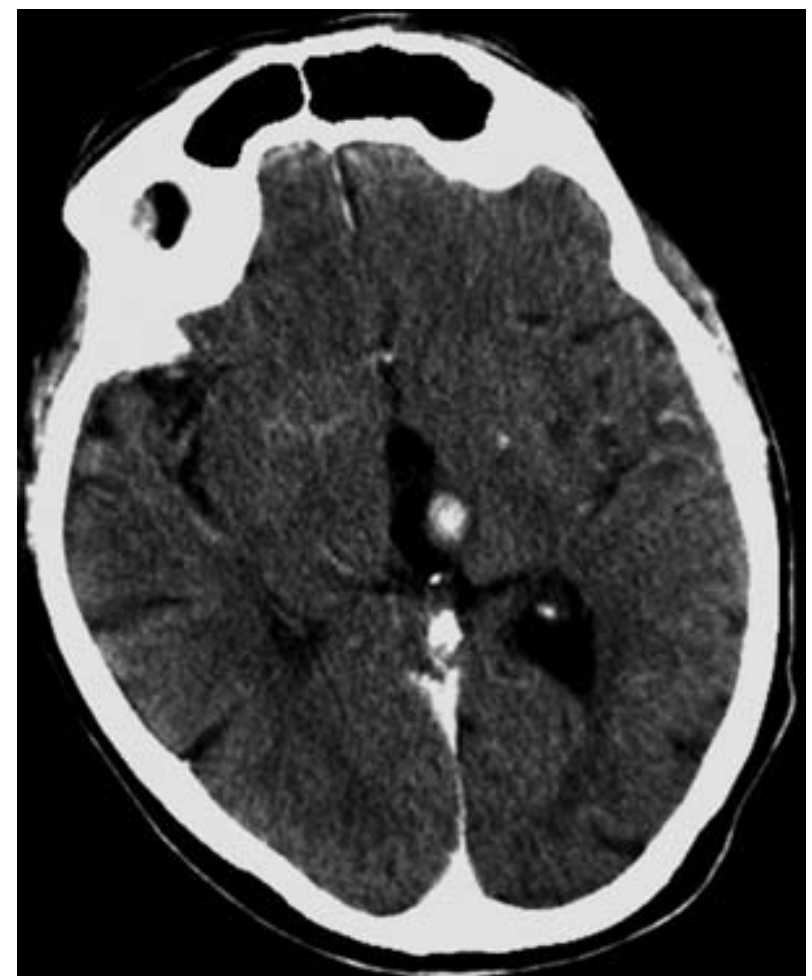

Figure 1: CT brain shows small left thalamic hemorrhage.

multiple foci of signal loss on the gradient-recalled echo (GRE) sequence, indicative of microbleeds, in the brainstem, thalamus, and the inferior periventricular white matter (Figure 2). There were no microbleeds in the more superior hemispheric white matter and corona radiata. There was evidence of white matter disease and microbleeds in the brainstem and cerebellar atrophy (Figure 3). Moderate leukoaraiosis (grade 2 using the Fazekas and Schmidt scale $)^{1}$ was present. An MRA showed no stenosis of

From the Department of Clinical Neurosciences (NS, EES, AMD, NUW); Hotchkiss Brain Institute (EES, AMD), Calgary, Alberta, Canada.

Received October 30, 2008. Final Revisions Submitted January 14, 2009. Correspondence to: Andrew M. Demchuk, Calgary Stroke Program, Department of Clinical Neurosciences, Room 1162, Foothills Medical Centre, 1403-29 Street NW, Calgary, Alberta, T2N 2T9, Canada. 


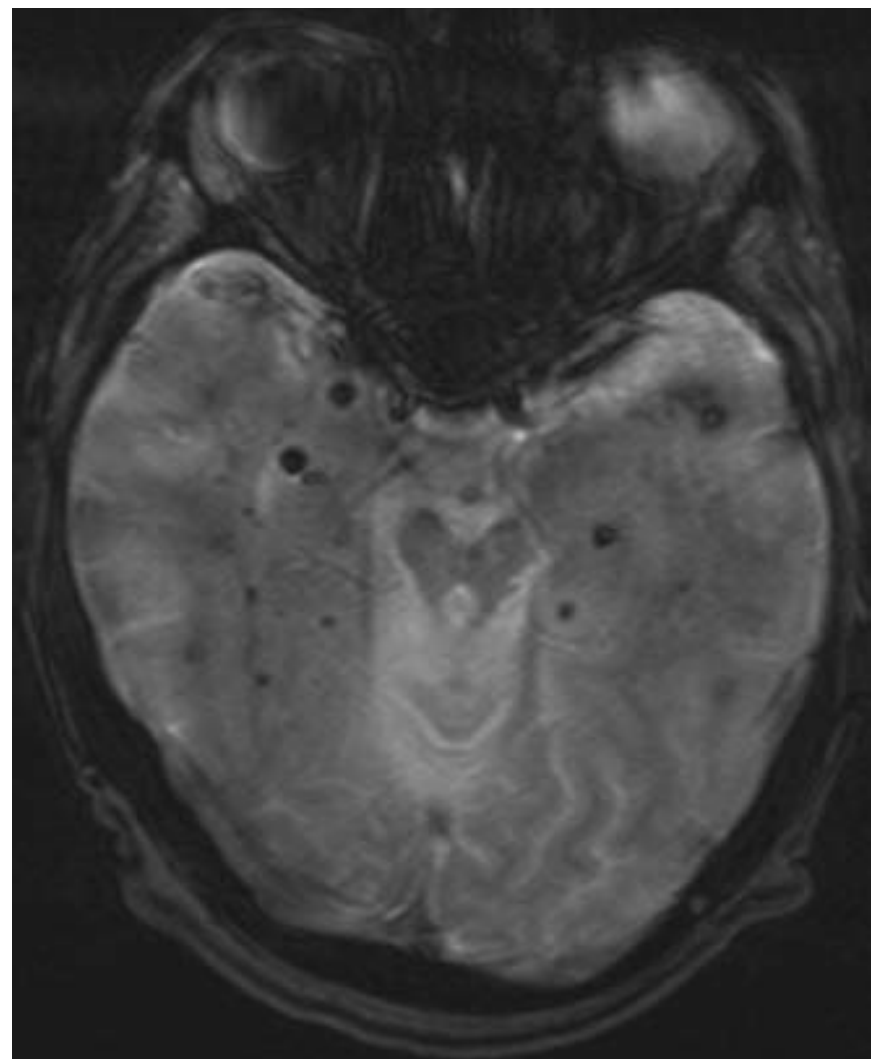

Figure 2: MRI gradient-recalled echo (GRE) sequence showing multiple microbleeds in the brainstem, thalamus, medial temporal lobes and the inferior periventricular white matter.

the major branches of the Circle of Willis or the extracranial carotid and vertebral arteries.

\section{DisCuSSION}

Our patient presented with recurrent episodes of brainstem stroke from the age of 37 years. The CT brain performed repeatedly showed only lacunar infarcts and a single episode of thalamic hemorrhage. The MRA did not demonstrate any stenotic lesion of the large vessels. The GRE sequence showed multiple microbleeds in the brainstem region which corresponded to the irradiated field. Cavernous angiomas are unlikely to be the cause of the hemorrhage and microbleeds in our case because none of the lesions displayed the characteristic imaging appearance of acute and chronic bleeding of various ages.

Irradiation leads to accelerated development of atherosclerosis in exposed large arteries by injury to the endothelial cells lining the lumen of the carotid artery ${ }^{2}$. It is more diffuse than traditional atherosclerosis ${ }^{3}$. The locations of atherosclerosis can vary but are usually confined to the extracranial vasculature. Intracranial atherosclerosis has also been reported $^{4}$. A single case of radiation induced lacunar syndrome has been described ${ }^{5}$. Cavernous angioma development

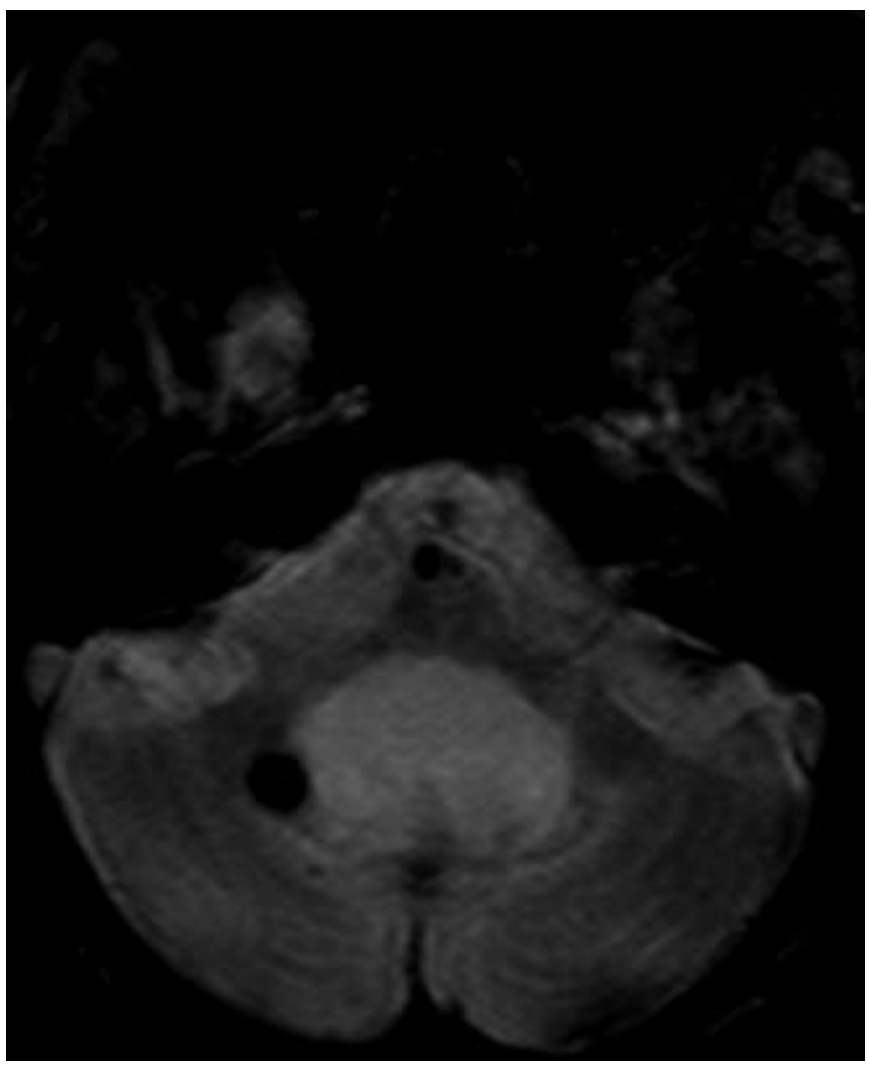

Figure 3: MRI gradient-recalled echo (GRE) sequence showing microbleeds, white matter disease of the brainstem, cerebellar atrophy at the level of 4 th ventricle.

has been reported after brain irradiation in pediatric brain tumours ${ }^{6}$.

The likely cause of the extensive symptomatic small vessel disease in this patient is radiation-induced injury. He did not have any significant vascular risk factors and his strokes began at a young age. Therefore the severity of his cerebrovascular disease is not accounted for by traditional risk factors. The absence of a family history of early-onset stroke or dementia argues against a genetic predisposition to stroke such as cerebral autosomal dominant arteriopathy with subcortical ischemic leukoencephalopathy (CADASIL).

Radiation injury to the small vessels can be seen pathologically, where radiation injures capillaries, sinusoids, and small arteries, in that order of severity ${ }^{7}$. Other symptoms in our case are also consistent with radiation injury. Radiation induced cerebral vasculopathy may present with headache or speech disturbance as the initial symptom ${ }^{8}$.Our patient had new onset recurrent headaches a decade after initiation of radiotherapy; also he had prominent dysarthria. He also had progressive hearing impairment presumably secondary to radiation injury.

A literature search did not yield similar cases of recurrent radiation-induced small vessel disease and strokes. To our knowledge, radiation vasculopathy presenting with recurrent 
ischemic strokes, microbleeds and symptomatic hemorrhage is novel and has not been previously reported.

In conclusion, strokes secondary to irradiation may occur long after radiotherapy. Although radiotherapy is most commonly linked with large vessel disease, our case suggests that small artery disease and hemorrhagic strokes may also be consequences of irradiation.

\section{REFERENCES}

1. Kapeller P, Barber R, Vermeulen RJ, Adèr H, Scheltens P, Freidl W, et al. Visual rating of age-related white matter changes on magnetic resonance imaging: scale comparison, interrater agreement, and correlations with quantitative measurements. Stroke. 2003; 34(2):441-5.

2. Lopez M, el-Bayar H, Hye RJ, Freischlag J. Carotid artery disease in patients with head and neck carcinoma. Am Surg. 1990; 56(12):778-81.

3. Eisenberg RL, Hedgcock MW, Wara WM, Jeffrey RB. Radiationinduced disease of the carotid artery. West J Med. 1978; 129(6): 500-3.
4. Kang JH, Kwon SU, Kim JS. Radiation-induced angiopathy in acute stroke patients. J Stroke Cerebrovasc Dis. 2002; 11(6):315-9.

5. Salih IS, Higgins NJ, Warburton EA, Baron JC. Lacunar stroke attributable to radiation-induced intracranial arteriopathy. Eur J Neurol. 2007 Aug;14(8):937-9.

6. Baumgartner JE, Ater JL, Ha CS, Kuttesch JF, Leeds NE, Fuller GN, et al. Pathologically proven cavernous angiomas of the brain following radiation therapy for pediatric brain tumors. Pediatr Neurosurg. 2003 Oct; 39(4):201-7.

7. Fajardo LF, Berthrong M. Vascular lesions following radiation. Pathol Annu. 1988; 23:297-330.

8. Bitzer M, Topka H. Progressive cerebral occlusive disease after radiation therapy. Stroke. 1995; 26(1):131-6. 